\title{
Effect of Application of Graded Level of Phosphorus to Finger Millet - Maize Cropping System in Soils of Different P Fertility
}

\author{
M. Chandrakala ${ }^{1 *}$, C.A. Srinivasamurthy ${ }^{2}$, Sanjeev Kumar ${ }^{3}$ and D.V. Naveen ${ }^{4}$ \\ ${ }^{1}$ National Bureau of Soil Survey and Land Use Planning, Regional Centre, \\ Hebbal, Bangalore-560 024, Karnataka, India \\ ${ }^{2}$ Director of Research, Central Agricultural University, Imphal, Manipur, India \\ ${ }^{3}$ National Dairy Research Institute, Karnal Haryana 132001 \\ ${ }^{4}$ Deptartment of Soil Science and Agricultural Chemistry, Sericulture College, \\ Chintamani, Karnataka, India \\ *Corresponding author
}

\begin{abstract}
A B S T R A C T
Fertilizers are one of the costly inputs but continue to exert significant contribution to produce additional food grains. To realize maximum benefits and reduce nutrient losses from fertilizers, they must be applied in the right quantity, source and combination at the right time using the right method. An investigation on requirement of $\mathrm{P}$ for finger millet and maize in EDZ of Karnataka was taken at UAS, Bangalore. Response of finger millet and maize to graded levels of $\mathrm{P}$ was studied in the field by creating five phosphorus fertility gradients viz., $\mathrm{P}_{0}$ (Very low), $\mathrm{P}_{1}$ (Low), $\mathrm{P}_{2}$ (Medium), $\mathrm{P}_{3}$ (High) and $\mathrm{P}_{4}$ (Very high) (< 15: 16-30: 31-45: 46-60: > $60 \mathrm{~kg} \mathrm{P}_{2} \mathrm{O}_{5} \mathrm{ha}^{-1}$, respectively). Results revealed that, application of $125 \%$ RDP with rec. NK\&FYM recorded higher finger millet (31.66 and $55.32 \mathrm{q} \mathrm{ha}^{-1}$ grain and straw, respectively) and maize (78.26 q ha ${ }^{-1}$ grain) yield in high $\mathrm{P}$ strip. Grain yield and harvest index of cropping system as a whole improved significantly in FYM supplied plots. Phosphorus use efficiency in maize was higher than finger millet. The higher use efficiency was recorded in very low gradient strip. Higher PUE among treatments was obtained when $\mathrm{P}$ was applied at the rate of $75 \%$ of recommended dose along with rec. N\&K. Application of $75 \%$ rec. $\mathrm{P}+$ rec. N\&K recorded higher B: C ratio in both the crops and was the best and optimum $\mathrm{P}$ prescription for these crops as the $\mathrm{P}$ use efficiency was also higher.
\end{abstract}

\section{Introduction}

The soil test ratings of low, medium and high fertility classes for nutrients are currently adopted by the soil testing laboratories need a fresh look as significant responses to applied nutrients have been recorded in soils with high fertility status in many crops. Phosphorus responses decrease with improvement in soil P-status, as expected. In
Punjab, responses were obtained upto 60-90 $\mathrm{kg} \mathrm{P}_{2} \mathrm{O}_{5} \mathrm{ha}^{-1}$ in low-P soils, upto $60 \mathrm{~kg} \mathrm{P}_{2} \mathrm{O}_{5}$ $\mathrm{ha}^{-1}$ in medium-P soil and there was little response to $\mathrm{P}$ in high-P soils. In alluvial soils of U.P., even on soils classified as high in available $\mathrm{P}, 60 \mathrm{~kg} \mathrm{P}_{2} \mathrm{O}_{5} \mathrm{ha}^{-1}$ increased the wheat yield by $653 \mathrm{~kg} \mathrm{ha}^{-1}$ or $10.9 \mathrm{~kg}$ grain per kg $\mathrm{P}_{2} \mathrm{O}_{5}$. 
Even at the current high price of $\mathrm{P}_{2} \mathrm{O}_{5}$, this underscores the need to revise soil fertility limits (Tiwari, 2002). If the initial soil phosphorus level is high, then maintenance application will be enough (Anonymous, 2012).

Finger millet [Eleusine coracana (L.) Gaertn.] [F: Poaceae] also known as Ragi or African millet ranks fourth in importance among millets in the world after sorghum (Sorghum bicolor), pearl millet (Pennisetum glaucum) and foxtail millet (Setaria italica). Of the total area of 2.7 mha under millets, Ragi alone accounts for 1.6 mha of the area and 75 per cent of total production in the country. India is the world's largest producer with annual production of $2.1 \mathrm{mt}$ and productivity of around $1300 \mathrm{~kg} \mathrm{ha}^{-1}$. In India, ragi ranks fourth among the grain crops in productivity after wheat, rice and maize. Under irrigated conditions, the crop has a yield potential of 3 $4 \mathrm{t} \mathrm{ha}^{-1}$ (Anonymous, 2009).

Maize (Zea mays L.) is one of the important cereals cultivated in India and it ranks fourth after rice, wheat and sorghum. Maize area in Karnataka was insignificant during the pregreen revolution years. The yield levels were also low in the range of $700-900 \mathrm{~kg} \mathrm{ha}^{-1}$. The maize area in the state increased steadily because of the steady high yield levels and assured market prices. The present area under cultivation in the state is 10.7 lakh ha with a production of 30.3 lakh tons and a productivity of $2833 \mathrm{~kg} \mathrm{ha}^{-1}$ in $2008-09$.

In the light of the above facts, a field experiment involving gradient creation followed by response of crops with graded levels of phosphorus along with other recommended dose of nutrients and FYM as organic source was conducted at the Zonal Agricultural Research Station, University of Agricultural Sciences, GKVK during 20092012, with the objective is to study the effect of application of graded levels of phosphorus applied to soils with different fertility gradient on growth of finger millet and maize.

\section{Materials and Methods}

The field experiment comprised of two stages. Fertility gradient creation was the preparatory step as per the procedure of Ramamoorthy et al., (1967) followed by finger millet-maize cropping system in the subsequent seasons. The experiment was conducted at D-16 Block, Zonal Agricultural Research Station (ZARS), GKVK, UAS, Bengaluru which is located in Eastern Dry Zone of Karnataka Surface soil $(0-15 \mathrm{~cm})$ was analyzed for physical and chemical properties by adopting standard procedures. The soil of experimental site was red sandy clay loam in texture, acidic in reaction, low in available nitrogen (203.84 $\mathrm{kg} \mathrm{ha}^{-1}$ ) and phosphorus (18.42 $\mathrm{kg} \mathrm{ha}^{-1}$ ) and medium in available potassium $(147.12 \mathrm{~kg}$ ha $^{-1}$ ) content.

Five equal strips $\left(45 \times 8.2 \mathrm{~m}^{2}\right)$ were created in one and the same field and named very low (VL), low (L), medium (M), high (H) and very high $(\mathrm{VH})$ gradient strips as $\mathrm{P}_{0}, \mathrm{P}_{1}, \mathrm{P}_{2}, \mathrm{P}_{3}$ and $\mathrm{P}_{4}$, respectively. Graded doses of phosphorus viz. $0,20,40,80$ and $120 \mathrm{~kg} \mathrm{ha}^{-1}$ was applied through fertilizer and organics 50 per cent each so as to achieve Very low $(<15$ $\left.\mathrm{kg} \mathrm{P}_{2} \mathrm{O}_{5} \mathrm{ha}^{-1}\right)$, Low (16-30 kg $\mathrm{P}_{2} \mathrm{O}_{5} \mathrm{ha}^{-1}$ ), Medium (31- $45 \mathrm{~kg} \mathrm{P}_{2} \mathrm{O}_{5} \mathrm{ha}^{-1}$ ), High (46 - 60 $\mathrm{kg}_{2} \mathrm{O}_{5} \mathrm{ha}^{-1}$ ) and Very high (> $60 \mathrm{~kg} \mathrm{P}_{2} \mathrm{O}_{5}$ $\left.\mathrm{ha}^{-1}\right) \mathrm{P}$ levels in the respective strips. Exhaustive crop fodder maize was grown and green fodder was harvested at 60 days after sowing. Soils in each strip analyzed for available nutrients status. Available $\mathrm{P}_{2} \mathrm{O}_{5}$ content obtained in $\mathrm{P}_{0}, \mathrm{P}_{1}, \mathrm{P}_{2}, \mathrm{P}_{3}$ and $\mathrm{P}_{4}$, was $14.82,27.37,38.76,52.25,80.72 \mathrm{~kg} \mathrm{ha}^{-1}$, respectively.

Finger millet (GPU-28) was grown during summer followed by maize was grown during 
kharif 2011 by imposing treatments in a factorial RCBD design. Treatment details as follows; $\mathrm{T}_{1}$ : Absolute control; $\mathrm{T}_{2}$ : Package of Practice $(\mathrm{NPK}+\mathrm{FYM}) ; \mathrm{T}_{3}: 100 \%$ Rec. N, P \&K only (no FYM); $\mathrm{T}_{4}: 75 \%$ Rec. $\mathrm{P}+$ rec. dose of N\&K (no FYM); $\mathrm{T}_{5}: 75 \%$ Rec. $\mathrm{P}+$ Rec. dose of N\&K only+ Rec. FYM; T $: 125$ $\%$ Rec. P + Rec. dose of N\&K (no FYM); $\mathrm{T}_{7}$ : $125 \%$ Rec. P + Rec. dose of N\&K + Rec. FYM.

The ragi ear heads harvested from each plot were sun dried and threshing was done to separate out the grains. Grain weight was recorded and grain yield in quintal per ha was calculated. For maize, the net plot was marked, cobs were harvested separately and total dried cob yield from each net plot was recorded. After threshing, grains were separated, cleaned and grain weight was recorded in quintal per ha. Finger millet straw was hand reaped from each plot, sun dried and dry weight was recorded and was calculated in quintal per hectare. Maize stover yield per net plot was recorded for each treatment. Later the stover yield per net plot was computed on hectare basis and then it was converted in to quintal per ha.

Phosphorus Use Efficiency (PUE) defined as 'yield per unit of nutrient supplied (from the soil and or fertilizer)' which was determined by using the formula of Moll et al., (1982). The harvest index (HI) was calculated as the ratio of grain weight to total above ground crop dry weight as per Terao et al., (2011). Cost of cultivation was calculated based on the prices of inputs that were prevailing in the market at the time of their use. The selling price for the produce was obtained from APMC market, Bangalore. The net returns per hectare was calculated by deducting the cost of cultivation from gross income and expressed in rupees per hectare (Rs. ha ${ }^{-1}$ ).

The analysis and interpretation of the data were done using the Fisher's method of analysis and variance technique. For field experiment data were analyzed using ANOVA (Two-Way) with interaction effect. Critical differences among $\mathrm{P}$ fertility gradient strips, treatments and interactions were estimated at $5 \%$ probability level of significance (Panse and Sukhatme, 1985).

\section{Results and Discussion}

The results of the investigation on performance of crops on different phosphorus fertility gradient strips applied with graded levels of phosphorus to finger millet-maize cropping system are presented in this chapter under the following sub headings.

\section{Response of finger millet yield}

Finger millet grain and straw yield (Table 1) were significantly increased due to application of graded levels of phosphorus to different $\mathrm{P}$ fertility gradient strips. Yields increased as the $\mathrm{P}$ fertility gradient increased to high $\mathrm{P}$ fertility strips and then declined. Among the $\mathrm{P}$ fertility gradient strips, $\mathrm{P}_{3}$ (high P) strip recorded significantly higher mean grain and straw yield followed by medium fertility gradient strip compared to other fertility gradient strips. Low $\mathrm{P}$ gradient strip recorded significantly higher yields over very low strip and was on par with very high $\mathrm{P}$ fertility strip. However, very low gradient strip recorded lower grain and straw yield which may be due to the contribution of initial soil fertility and residual effect of organic manure (neem cake).

Higher yield in high P strip soils could be due to better bioavailability of $\mathrm{P}$ and adequate as well as balanced supply of all the essential nutrients. The magnitude of response to applied $\mathrm{P}$ decreased sharply with increase in soil available $\mathrm{P}$ status and also with increase in applied P levels. Dhillon et al., (1987) obtained similar results. In high $\mathrm{P}$ soils, the decrease in yield response per unit increase in 
applied $\mathrm{P}$ could possibly be attributed to higher $\mathrm{P}$ concentration in soil-plant system leading to antagonistic effect on availability of $\mathrm{Zn}$ to plants and also to the imbalance in nutrients availability and uptake. Lu Ru-Kun (1981) reported a positive response to $\mathrm{P}$ application and observed 17.0- $40.9 \mathrm{~kg}$ increase in rice yield per $\mathrm{kg}$ of $\mathrm{P}$ applied on $\mathrm{P}$ deficient soils of China. Hanumanthappa and Shivaraj (2003) observed that application of neem cake at $750 \mathrm{~kg} \mathrm{ha}^{-1}$ recorded the higher seed yield and stalk yield of sesamum which was significantly higher compared to plots not involving organic manures.

Yield reduction in very high fertility gradient strip may be attributed to excessive addition of $\mathrm{P}$ that predisposed the crop to pest and disease incidences which in turn affected the crop growth and productivity. Decrease in yield of crop might be due to lower uptake of all the essential nutrients and to imbalanced supply of nutrients through fixation and antagonism effect of $\mathrm{P}$ with $\mathrm{Ca}, \mathrm{Fe}$ and $\mathrm{Zn}$, respectively. Thus, once the soil test $\mathrm{P}$ level reached the optimum for crop yield, it was recommended that $\mathrm{P}$ fertilizer application be restricted or stopped to minimize negative effects. Field survey data of Zhang XiaoSheng et al., (2007) reported similar findings.

Among treatments, mean grain and straw yield were increased with the increased rate of phosphorus application along with FYM. Treatment involving $125 \%$ rec. $\mathrm{P}+$ rec. N\&K + rec. FYM $\left(\mathrm{T}_{7}\right)$ recorded significantly higher grain and straw yield followed by nutrient application as per package of practice $\left(\mathrm{T}_{2}\right)$. Treatments $\mathrm{T}_{5}(75 \%$ rec. $\mathrm{P}+$ rec. $\mathrm{N} \& \mathrm{~K}+$ rec. FYM) and $\mathrm{T}_{6}$ were significantly higher over $\mathrm{T}_{4}(75 \%$ rec. $\mathrm{P}+$ rec. $\mathrm{N} \& \mathrm{~K})$ and absolute control $\left(\mathrm{T}_{1}\right)$. Lower yield (11.38 and $25.09 \mathrm{q}$ $\mathrm{ha}^{-1}$, respectively) was recorded in absolute control $\left(\mathrm{T}_{1}\right)$. Phosphorus, being primary essential nutrient, has prime importance in crop nutrition. It is involved in almost all the biochemical pathways as a component of energy carrier compounds ATP and ADP. Hence, these results emphasize the importance of applying adequate $\mathrm{P}$ for consistently high yields. Khalil and Jan (2003) and Guo Jianhua et al., (2000) also got similar results.

Application of FYM along with 100 per cent recommended NPK recorded significantly higher mean finger millet grain yield compared to recommend NPK which may be due to buildup of soil phosphorus due to FYM application which also lowered the phosphorus fixation in Alfisols, thus making higher proportion of applied phosphorus available for crop utilization. Similar findings were reported by Thimma Reddy (1987) and Anon. (2000). Lower level of $\mathrm{P}$ application yielded the lower grain yield, whereas the higher fertilizer $\mathrm{P}$ treatment produced maximum yield. The grain yield increased significantly upto $62 \mathrm{~kg} \mathrm{P}_{2} \mathrm{O}_{5} \mathrm{ha}^{-1}$ in very low, low and medium $\mathrm{P}$ strip soil as compared to very high strip soils. There was a significant response to applied $\mathrm{P}$ in very low, low and medium $\mathrm{P}$ strips. However, application of higher rate of $\mathrm{P}$ in very high $\mathrm{P}$ strip showed only marginal increase in yield. Dhillon et al., (1987) reported similar responses for $\mathrm{P}$ application.

Application of $75 \%$ rec. $\mathrm{P}+$ Rec. N\&K recorded significantly higher yield over absolute control and was on par with 100 percent RDF which could be due to better $\mathrm{P}$ utilization in these two treatments. Ghanshyam et al., (2010), Niazi et al., (1990) and Khan et al., (2000) reported increased grain yield due to $\mathrm{P}$ application over control. More and Agale (1993) noticed increased seed cotton and dry matter yield with increasing level of $\mathrm{P}$ upto $50 \mathrm{~kg} \mathrm{P}_{2} \mathrm{O}_{5} \mathrm{ha}^{-1}$. Ananda (2009) reported significantly higher grain and straw yield of finger millet over control with application of 100 per cent NPK + FYM in finger millet-soybean cropping system. 
Application of RDF $100 \%+$ FYM recorded significantly higher yield over $125 \%$ rec. $\mathrm{P}+$ rec. N\&K. Similar findings were reported by Virupakshi (1988). However, $100 \%$ RDF + FYM also recorded significantly higher yield over $100 \%$ RDF due to considerable increase in the organic carbon content and a marginal increase in cation exchange capacity of soil (Anon., 1996). Nimje and Seth (1988) reported higher yield of soybean with increasing rates of $\mathrm{P}_{2} \mathrm{O}_{5}\left(0,40\right.$ and $\left.80 \mathrm{~kg} \mathrm{ha}^{-1}\right)$ along with farmyard manure. Subramanian and Kumaraswamy (1989) recorded higher dry matter production of finger millet under 100 per cent NPK + FYM @ 10 t per ha under finger millet-cowpea-maize cropping sequence.

Judicious use of chemical fertilizers and organic manure would lead to efficient use of fertilizer and integrated nutrient management supply system and management holds a great promise in reducing the fertilizer dose for crop production. Higher yield can be produced by distributing fertilizer based on initial soil test values. These findings are in accordance with Prasad and Prasad (1994) and Prasad et al., (1984). Balasubramaniyan (2005) reported that combined application of FYM with $\mathrm{P}$ fertilizer resulted in higher rice grain yield.

Interaction effect showed significantly higher finger millet grain and straw yield in high $\mathrm{p}$ strip $\left(\mathrm{P}_{3}\right)$ with treatment $\mathrm{T}_{7}$ which received $125 \%$ rec. $\mathrm{P}+$ rec. $\mathrm{N} \& \mathrm{~K}+$ rec. $\mathrm{FYM}$ followed by treatment $\mathrm{T}_{2}$ (Pop). Lower yield was recorded in very low $\mathrm{P}$ gradient strip with absolute control.

The response to applied $\mathrm{P}$ was more pronounced on soils low in available phosphorus than in other soils. In general, the response to $\mathrm{P}$ was very sharp in soils having low extractable P. Gradual increase in yield was observed in very low, low, medium and high available $\mathrm{P}$ soils at all the $\mathrm{P}$ levels and was found to be statistically significant. These findings are confirming the result of Ali and Rahman (2011) and Muralidharudu et al., (2003).

\section{Response of maize yield}

Numerous studies have shown that maize crop responded to fertility levels under various edapho-climatic conditions. Response to added fertilizer is dependent upon the variety, initial fertility status of soil and climatic conditions. With the advent of high yielding varieties and hybrids and pressing demand to increase the quality, adequate application of fertilizers is becoming increasingly important.

Maize grain and stover yields were increased with the increased $\mathrm{P}$ gradients from very low $\left(\mathrm{P}_{0}\right)$ to high $\mathrm{P}\left(\mathrm{P}_{3}\right)$ gradient strip and were decreased as the gradient increased to very high (Table 2). High $\mathrm{P}$ gradient strip recorded significantly higher yield followed by medium fertility gradient strip which could be due to better root growth and development with $\mathrm{P}$ fertilization encouraged the higher uptake of nutrients resulting in better grain formation and also due to residual effect of organics and inorganics applied to previous finger millet crop. Low, medium, high and very high strips were on par with each other with respect to stover yield.

This may be due to better utilization of nutrients in all the gradient strips. Very low strip $\left(\mathrm{P}_{0}\right)$ recorded lower yield which may be due to inadequate supply of nutrients as the maize is an exhaustive crop. Hence, properly managed FYM and NP fertilizers could be used for maize production. In areas of marginal $\mathrm{P}$ deficiency, application of $\mathrm{P}$ fertilizers is essential to marginal higher yields under intensive cropping. Datta and Gomez (1975) reported the similar findings. 
Table.1 Grain and straw yield $\left(\mathrm{q} \mathrm{ha}^{-1}\right)$ of finger millet grown on different phosphorus fertility gradient strips as influenced by graded levels of applied phosphorus to finger millet-maize cropping system

\begin{tabular}{|c|c|c|c|c|c|c|c|c|c|c|c|c|}
\hline \multirow{2}{*}{$\begin{array}{c}\text { P levels/ } \\
\text { Treatments }\end{array}$} & \multicolumn{6}{|c|}{ Grain } & \multicolumn{6}{|c|}{ Straw } \\
\hline & $\mathbf{P}_{\mathbf{0}}$ & $\mathbf{P}_{1}$ & $\mathbf{P}_{2}$ & $\mathbf{P}_{3}$ & $\mathbf{P}_{4}$ & Mean & $\mathbf{P}_{0}$ & $\mathbf{P}_{1}$ & $\mathbf{P}_{2}$ & $\mathbf{P}_{3}$ & $\mathbf{P}_{4}$ & Mean \\
\hline $\mathbf{T}_{1}$ & 8.51 & 9.81 & 10.73 & 12.88 & 14.97 & 11.38 & 21.68 & 23.80 & 25.89 & 26.92 & 27.14 & 25.09 \\
\hline $\mathbf{T}_{2}$ & 25.70 & 26.32 & 28.86 & 29.53 & 24.88 & 27.06 & 49.02 & 51.32 & 53.67 & 54.33 & 47.26 & 51.12 \\
\hline $\mathbf{T}_{3}$ & 22.55 & 25.21 & 26.35 & 26.79 & 24.28 & 25.04 & 45.55 & 49.51 & 49.89 & 50.13 & 48.28 & 48.67 \\
\hline $\mathbf{T}_{4}$ & 20.89 & 24.20 & 25.54 & 25.88 & 24.06 & 24.12 & 43.56 & 47.53 & 49.29 & 48.88 & 48.06 & 47.47 \\
\hline $\mathbf{T}_{5}$ & 23.60 & 25.27 & 28.29 & 29.31 & 24.17 & 26.13 & 46.93 & 48.32 & 51.95 & 52.95 & 47.42 & 49.52 \\
\hline $\mathbf{T}_{6}$ & 24.68 & 24.77 & 27.10 & 27.87 & 23.80 & 25.65 & 48.15 & 47.78 & 50.60 & 51.21 & 48.80 & 49.31 \\
\hline $\mathbf{T}_{7}$ & 26.66 & 28.29 & 30.08 & 31.66 & 24.05 & 28.15 & 50.32 & 52.29 & 51.08 & 55.32 & 47.39 & 51.28 \\
\hline \multirow[t]{2}{*}{ Mean } & 21.80 & 23.41 & 25.28 & 26.28 & 22.89 & 23.93 & 43.60 & 45.79 & 47.48 & 48.54 & 44.91 & 46.06 \\
\hline & $\mathbf{F}$ & S.Em \pm & \multicolumn{2}{|c|}{$\mathrm{CD}(p=0.05)$} & $\mathbf{C V}$ & & $\mathbf{F}$ & S.Em \pm & \multicolumn{2}{|c|}{$\mathrm{CD}(p=0.05)$} & $\mathbf{C V}$ & \\
\hline $\mathbf{P}$ & $\mathrm{S}$ & 0.31 & \multicolumn{2}{|c|}{0.86} & \multirow{3}{*}{5.85} & & $\mathrm{~S}$ & 0.399 & \multicolumn{2}{|c|}{1.13} & \multirow{3}{*}{5.98} & \\
\hline $\mathbf{T}$ & $\mathrm{S}$ & 0.36 & \multicolumn{2}{|c|}{1.02} & & & $\mathrm{~S}$ & 0.47 & \multicolumn{2}{|c|}{1.34} & & \\
\hline $\mathbf{P} \times \mathbf{T}$ & $\mathrm{S}$ & 0.81 & \multicolumn{2}{|c|}{2.28} & & & $\mathrm{~S}$ & 1.06 & \multicolumn{2}{|c|}{2.98} & & \\
\hline \multicolumn{7}{|c|}{$\begin{array}{l}\mathrm{T}_{1}: \text { Absolute control } \\
\mathrm{T}_{2}: \text { Package of Practice (rec. NPK+FYM) } \\
\mathrm{T}_{3}: 100 \text { per cent rec. } \mathrm{N}, \mathrm{P} \& \mathrm{~K} \text { (no FYM) } \\
\mathrm{T}_{4}: 75 \text { per cent rec. } \mathrm{P}+\text { rec. } \mathrm{N} \& \mathrm{~K} \text { (no FYM) } \\
\mathrm{T}_{5}: 75 \text { per cent rec. } \mathrm{P}+\text { rec. } \mathrm{N} \& \mathrm{~K}+\text { rec. FYM } \\
\mathrm{T}_{6}: 125 \text { per cent rec. } \mathrm{P}+\text { rec. } \mathrm{N} \& \mathrm{~K} \text { (no FYM) }\end{array}$} & $\begin{array}{l}\text { Very lov } \\
\text { Low Pho } \\
\text { Medium } \\
\text { High Ph } \\
\text { Very hig }\end{array}$ & $\begin{array}{l}\text { hosphorus } \\
\text { horus fertil } \\
\text { losphorus } \mathrm{f} \\
\text { horus ferti } \\
\text { hosphorus }\end{array}$ & $\begin{array}{l}\text { tility stri } \\
\text { strip } \\
\text { ility strip } \\
\text { strip } \\
\text { rtility str }\end{array}$ & & & \\
\hline
\end{tabular}


Table. 2 Grain and stover yield ( $\mathrm{q} \mathrm{ha}^{-1}$ ) of maize grown on different phosphorus fertility gradient strips as influenced by graded levels of applied phosphorus to finger millet-maize cropping system

\begin{tabular}{|c|c|c|c|c|c|c|c|c|c|c|c|c|}
\hline \multirow{2}{*}{$\begin{array}{c}\text { P levels/ } \\
\text { Treatments }\end{array}$} & \multicolumn{6}{|c|}{ Grain } & \multicolumn{6}{|c|}{ Stover } \\
\hline & $\mathbf{P}_{\mathbf{0}}$ & $\mathbf{P}_{1}$ & $\mathbf{P}_{2}$ & $\mathbf{P}_{3}$ & $\mathbf{P}_{4}$ & Mean & $\mathbf{P}_{0}$ & $\mathbf{P}_{1}$ & $\mathbf{P}_{2}$ & $\mathbf{P}_{3}$ & $\mathbf{P}_{4}$ & Mean \\
\hline $\mathbf{T}_{1}$ & 15.00 & 22.65 & 27.22 & 28.78 & 30.67 & 24.86 & 27.93 & 40.08 & 46.78 & 48.06 & 47.41 & 42.05 \\
\hline $\mathbf{T}_{2}$ & 63.72 & 70.66 & 74.48 & 76.43 & 71.80 & 71.42 & 104.28 & 109.73 & 109.83 & 115.13 & 109.21 & 109.64 \\
\hline $\mathbf{T}_{\mathbf{3}}$ & 54.74 & 65.15 & 69.03 & 70.33 & 66.92 & 65.23 & 91.76 & 110.77 & 111.80 & 114.63 & 108.80 & 107.55 \\
\hline $\mathbf{T}_{4}$ & 51.97 & 63.33 & 68.24 & 69.27 & 68.56 & 64.28 & 89.97 & 110.21 & 111.08 & 113.19 & 112.47 & 107.38 \\
\hline $\mathbf{T}_{\mathbf{5}}$ & 59.33 & 67.40 & 72.96 & 73.31 & 70.19 & 68.64 & 100.63 & 111.08 & 115.40 & 115.08 & 108.09 & 110.06 \\
\hline $\mathbf{T}_{6}$ & 59.72 & 66.85 & 70.58 & 71.57 & 62.63 & 66.27 & 95.52 & 111.66 & 109.54 & 110.51 & 105.66 & 106.58 \\
\hline $\mathbf{T}_{7}$ & 67.93 & 73.06 & 75.95 & 78.26 & 71.40 & 73.32 & 100.50 & 113.52 & 107.88 & 108.39 & 116.49 & 109.36 \\
\hline \multirow[t]{2}{*}{ Mean } & 53.20 & 61.30 & 65.49 & 66.85 & 63.17 & 62.00 & 87.23 & 101.01 & 101.76 & 103.57 & 101.16 & 98.95 \\
\hline & $\mathbf{F}$ & \multicolumn{2}{|c|}{ S.Em \pm} & \multicolumn{2}{|c|}{$C D(p=0.05)$} & $\mathbf{C V}$ & $\mathbf{F}$ & \multicolumn{2}{|c|}{ S.Em $E$} & \multicolumn{2}{|c|}{$C D(p=0.05)$} & $\mathrm{CV}$ \\
\hline $\mathbf{P}$ & $\mathrm{S}$ & \multicolumn{2}{|c|}{0.37} & \multicolumn{2}{|c|}{1.04} & & $\mathrm{~S}$ & \multicolumn{2}{|c|}{0.92} & \multicolumn{2}{|c|}{2.59} & \\
\hline $\mathbf{T}$ & $\mathrm{S}$ & \multicolumn{2}{|c|}{0.44} & \multicolumn{2}{|c|}{1.24} & 2.74 & $\mathrm{~S}$ & \multicolumn{2}{|c|}{1.09} & \multicolumn{2}{|c|}{3.06} & 4.25 \\
\hline $\mathbf{P} \times \mathbf{T}$ & $\mathrm{S}$ & \multicolumn{2}{|c|}{0.98} & \multicolumn{2}{|c|}{2.76} & & $\mathrm{~S}$ & \multicolumn{2}{|c|}{2.43} & \multicolumn{2}{|c|}{6.85} & \\
\hline \multicolumn{7}{|c|}{$\begin{array}{l}\mathrm{T}_{1}: \text { Absolute control } \\
\mathrm{T}_{2}: \text { Package of Practice (rec. NPK+FYM) } \\
\mathrm{T}_{3}: 100 \text { per cent rec. } \mathrm{N}, \mathrm{P} \& \mathrm{~K}(\text { no FYM) } \\
\mathrm{T}_{4}: 75 \text { per cent rec. } \mathrm{P}+\text { rec. } \mathrm{N} \& \mathrm{~K} \text { (no FYM) } \\
\mathrm{T}_{5}: 75 \text { per cent rec. } \mathrm{P}+\text { rec. } \mathrm{N} \& \mathrm{~K}+\text { rec. } \mathrm{FYM} \\
\left.\mathrm{T}_{6}: 125 \text { per cent rec. } \mathrm{P}+\text { rec. } \mathrm{N} \& \mathrm{~K} \text { (no } \mathrm{FYM}\right)\end{array}$} & $\begin{array}{l}\mathrm{P}_{0}: \text { Very } \\
\mathrm{P}_{1}: \text { Low } \\
\mathrm{P}_{2}: \text { Med } \\
\mathrm{P}_{3}: \text { High } \\
\mathrm{P}_{4}: \text { Very }\end{array}$ & $\begin{array}{l}\text { Ww Phosph } \\
\text { hosphorus } \\
\text { m Phospho } \\
\text { hosphorus } \\
\text { igh Phospl }\end{array}$ & $\begin{array}{l}\text { us fertility } \\
\text { tility strip } \\
\text { s fertility } \\
\text { rtility strip } \\
\text { us fertility }\end{array}$ & & & \\
\hline
\end{tabular}


Table.3 Harvest index for finger millet and maize as influenced by different phosphorus fertility gradient strips applied with graded levels of phosphorus to finger millet-maize cropping system

\begin{tabular}{|c|c|c|c|c|c|c|c|c|c|c|c|c|}
\hline \multirow{2}{*}{$\begin{array}{c}\text { P levels/ } \\
\text { Treatments }\end{array}$} & \multicolumn{6}{|c|}{ Finger millet } & \multicolumn{6}{|c|}{ Maize } \\
\hline & $\mathbf{P}_{0}$ & $\mathbf{P}_{1}$ & $\mathbf{P}_{2}$ & $\mathbf{P}_{3}$ & $\mathbf{P}_{4}$ & Mean & $\mathbf{P}_{\mathbf{0}}$ & $\mathbf{P}_{1}$ & $\mathbf{P}_{2}$ & $\mathbf{P}_{3}$ & $\mathbf{P}_{4}$ & Mean \\
\hline $\mathbf{T}_{1}$ & 0.28 & 0.29 & 0.29 & 0.32 & 0.36 & 0.31 & 0.35 & 0.36 & 0.37 & 0.37 & 0.39 & 0.37 \\
\hline $\mathbf{T}_{2}$ & 0.34 & 0.34 & 0.35 & 0.35 & 0.35 & 0.35 & 0.38 & 0.39 & 0.40 & 0.40 & 0.40 & 0.39 \\
\hline $\mathbf{T}_{\mathbf{3}}$ & 0.33 & 0.34 & 0.35 & 0.35 & 0.33 & 0.34 & 0.37 & 0.37 & 0.38 & 0.38 & 0.38 & 0.38 \\
\hline $\mathbf{T}_{4}$ & 0.32 & 0.34 & 0.34 & 0.35 & 0.33 & 0.34 & 0.37 & 0.36 & 0.38 & 0.38 & 0.38 & 0.37 \\
\hline $\mathbf{T}_{\mathbf{5}}$ & 0.33 & 0.34 & 0.35 & 0.36 & 0.34 & 0.34 & 0.37 & 0.38 & 0.39 & 0.39 & 0.39 & 0.38 \\
\hline $\mathbf{T}_{6}$ & 0.34 & 0.34 & 0.35 & 0.35 & 0.33 & 0.34 & 0.39 & 0.37 & 0.39 & 0.39 & 0.37 & 0.38 \\
\hline $\mathbf{T}_{7}$ & 0.35 & 0.35 & 0.37 & 0.36 & 0.34 & 0.35 & 0.40 & 0.39 & 0.41 & 0.42 & 0.38 & 0.40 \\
\hline \multirow[t]{2}{*}{ Mean } & 0.33 & 0.33 & 0.34 & 0.35 & 0.34 & 0.34 & 0.38 & 0.38 & 0.39 & 0.39 & 0.38 & 0.38 \\
\hline & $\mathbf{F}$ & \multicolumn{2}{|c|}{ S.Em \pm} & \multicolumn{2}{|c|}{$\mathrm{CD}(p=0.05)$} & $\mathbf{C V}$ & $\mathbf{F}$ & \multicolumn{2}{|c|}{ S.Em \pm} & \multicolumn{2}{|c|}{$\mathrm{CD}(p=0.05)$} & $\mathrm{CV}$ \\
\hline $\mathbf{P}$ & S & \multicolumn{2}{|c|}{0.003} & \multicolumn{2}{|c|}{0.007} & & $S$ & \multicolumn{2}{|c|}{0.002} & \multicolumn{2}{|c|}{0.006} & \\
\hline $\mathbf{T}$ & $S$ & \multicolumn{2}{|c|}{0.003} & \multicolumn{2}{|c|}{0.009} & 3.49 & S & \multicolumn{2}{|c|}{0.003} & \multicolumn{2}{|c|}{0.008} & 2.72 \\
\hline $\mathbf{P} \times \mathbf{T}$ & $S$ & \multicolumn{2}{|c|}{0.007} & \multicolumn{2}{|c|}{0.019} & & $S$ & \multicolumn{2}{|c|}{0.006} & \multicolumn{2}{|c|}{0.017} & \\
\hline \multicolumn{6}{|c|}{$\begin{array}{l}\mathrm{T}_{1}: \text { Absolute control } \\
\mathrm{T}_{2}: \text { Package of Practice (rec. NPK+FYM) } \\
\mathrm{T}_{3}: 100 \text { per cent rec. } \mathrm{N}, \mathrm{P} \& \mathrm{~K} \text { (no FYM) } \\
\mathrm{T}_{4}: 75 \text { per cent rec. } \mathrm{P}+\text { rec. } \mathrm{N} \& \mathrm{~K} \text { (no FYM) } \\
\mathrm{T}_{5}: 75 \text { per cent rec. } \mathrm{P}+\text { rec. } \mathrm{N} \& \mathrm{~K}+\text { rec. } \mathrm{FYM} \\
\mathrm{T}_{6}: 125 \text { per cent rec. } \mathrm{P}+\text { rec. } \mathrm{N} \& \mathrm{~K} \text { (no FYM) } \\
\mathrm{T}_{7}: 125 \text { per cent rec. } \mathrm{P}+\text { rec. } \mathrm{N} \& \mathrm{~K}+\text { rec. } \mathrm{FYM}\end{array}$} & $\begin{array}{l}\mathrm{P}_{0}: \\
\mathrm{P}_{1}: \\
\mathrm{P}_{2}: \\
\mathrm{P}_{3}: \\
\mathrm{P}_{4}:\end{array}$ & $\begin{array}{l}\text { ry low } \\
\text { w Phos } \\
\text { edium F } \\
\text { gh Phos } \\
\text { ry high }\end{array}$ & $\begin{array}{l}\text { phorus } \\
\text { us fertil } \\
\text { ohorus } f \\
\text { rus ferti } \\
\text { sphorus }\end{array}$ & $\begin{array}{l}\text { ility stri } \\
\text { strip } \\
\text { lity strif } \\
\text { strip } \\
\text { tility str }\end{array}$ & & & \\
\hline
\end{tabular}


Table.4 PUEP (Phosphorus Use Efficiency Per cent) by finger millet and maize grown on different phosphorus fertility gradient strips applied with graded levels of phosphorus to finger millet-maize cropping system

\begin{tabular}{|c|c|c|c|c|c|c|c|c|c|c|c|c|}
\hline \multirow{2}{*}{$\begin{array}{c}\text { P levels/ } \\
\text { Treatments }\end{array}$} & \multicolumn{6}{|c|}{ Finger millet } & \multicolumn{6}{|c|}{ Maize } \\
\hline & $\mathbf{P}_{\mathbf{0}}$ & $\mathbf{P}_{1}$ & $\mathbf{P}_{2}$ & $\mathbf{P}_{3}$ & $\mathbf{P}_{4}$ & Mean & $\mathbf{P}_{\mathbf{0}}$ & $\mathbf{P}_{1}$ & $\mathbf{P}_{2}$ & $\mathbf{P}_{3}$ & $\mathbf{P}_{4}$ & Mean \\
\hline $\mathbf{T}_{1}$ & 0.00 & 0.00 & 0.00 & 0.00 & 0.00 & 0.00 & 0.00 & 0.00 & 0.00 & 0.00 & 0.00 & 0.00 \\
\hline $\mathbf{T}_{2}$ & 31.24 & 28.25 & 27.36 & 24.11 & 15.85 & 25.36 & 38.40 & 38.89 & 39.13 & 42.85 & 38.00 & 39.45 \\
\hline $\mathbf{T}_{\mathbf{3}}$ & 31.56 & 30.95 & 26.51 & 24.09 & 15.41 & 25.70 & 36.93 & 42.88 & 40.16 & 44.86 & 38.29 & 40.62 \\
\hline $\mathbf{T}_{4}$ & 33.74 & 30.00 & 27.79 & 24.18 & 17.49 & 26.64 & 37.55 & 44.30 & 43.61 & 48.20 & 44.35 & 43.60 \\
\hline $\mathbf{T}_{5}$ & 31.75 & 28.12 & 28.00 & 24.68 & 16.18 & 25.75 & 36.93 & 42.36 & 43.92 & 45.08 & 40.43 & 41.74 \\
\hline $\mathbf{T}_{6}$ & 36.55 & 29.86 & 27.40 & 23.65 & 15.77 & 26.65 & 38.99 & 44.36 & 42.29 & 43.53 & 34.55 & 40.74 \\
\hline $\mathbf{T}_{7}$ & 31.48 & 29.56 & 26.55 & 25.97 & 15.44 & 25.80 & 36.83 & 39.54 & 39.37 & 39.30 & 35.59 & 38.13 \\
\hline Mean & 32.72 & 29.46 & 27.27 & 24.44 & 16.02 & 25.98 & 37.61 & 42.05 & 41.41 & 43.97 & 38.53 & 40.72 \\
\hline
\end{tabular}

$\mathrm{T}_{1}$ : Absolute control

$\mathrm{T}_{2}$ : Package of Practice (rec. NPK+FYM)

$\mathrm{T}_{3}: 100$ per cent rec. $\mathrm{N}, \mathrm{P} \& \mathrm{~K}$ (no FYM)

$\mathrm{T}_{4}: 75$ per cent rec. $\mathrm{P}+$ rec. $\mathrm{N} \& \mathrm{~K}($ no $\mathrm{FYM})$

$\mathrm{T}_{5}: 75$ per cent rec. $\mathrm{P}+$ rec. $\mathrm{N} \& \mathrm{~K}+$ rec. $\mathrm{FYM}$

$\mathrm{T}_{6}: 125$ per cent rec. $\mathrm{P}+$ rec. $\mathrm{N} \& \mathrm{~K}$ (no FYM)

$\mathrm{T}_{7}: 125$ per cent rec. $\mathrm{P}+$ rec. $\mathrm{N} \& \mathrm{~K}+$ rec. $\mathrm{FYM}$
$\mathrm{P}_{0}$ : Very low Phosphorus fertility strip

$\mathrm{P}_{1}$ : Low Phosphorus fertility strip

$\mathrm{P}_{2}$ : Medium Phosphorus fertility strip

$\mathrm{P}_{3}$ : High Phosphorus fertility strip

$\mathrm{P}_{4}$ : Very high Phosphorus fertility strip 
Table.5 Benefit cost analysis (B: C) of crops grown on different phosphorus fertility gradient strips applied with graded levels of phosphorus to finger millet-maize cropping system

\begin{tabular}{|c|c|c|c|c|c|c|c|c|c|c|c|c|}
\hline \multirow{2}{*}{$\begin{array}{c}\text { P levels/ } \\
\text { Treatments }\end{array}$} & \multicolumn{6}{|c|}{ Finger millet } & \multicolumn{6}{|c|}{ Maize } \\
\hline & $\mathbf{P}_{0}$ & $\mathbf{P}_{1}$ & $\mathbf{P}_{2}$ & $\mathbf{P}_{3}$ & $\mathbf{P}_{4}$ & Mean & $\mathbf{P}_{0}$ & $\mathbf{P}_{1}$ & $\mathbf{P}_{2}$ & $\mathbf{P}_{3}$ & $\mathbf{P}_{4}$ & Mean \\
\hline $\mathbf{T}_{1}$ & 1.88 & 2.14 & 2.34 & 2.73 & 3.09 & 1.88 & 1.59 & 2.38 & 2.85 & 3.01 & 3.18 & 2.60 \\
\hline $\mathbf{T}_{2}$ & 1.96 & 2.01 & 2.19 & 2.24 & 1.89 & 1.96 & 2.86 & 3.16 & 3.31 & 3.41 & 3.20 & 3.19 \\
\hline $\mathbf{T}_{\mathbf{3}}$ & 3.42 & 3.80 & 3.95 & 4.01 & 3.67 & 3.42 & 3.84 & 4.58 & 4.83 & 4.92 & 4.68 & 4.57 \\
\hline $\mathbf{T}_{4}$ & 3.33 & 3.81 & 4.01 & 4.05 & 3.80 & 3.33 & 3.77 & 4.60 & 4.93 & 5.00 & 4.95 & 4.65 \\
\hline $\mathbf{T}_{5}$ & 1.85 & 1.97 & 2.19 & 2.26 & 1.89 & 1.85 & 2.73 & 3.09 & 3.33 & 3.34 & 3.20 & 3.14 \\
\hline $\mathbf{T}_{6}$ & 3.56 & 3.57 & 3.88 & 3.98 & 3.47 & 3.56 & 4.05 & 4.55 & 4.77 & 4.83 & 4.26 & 4.49 \\
\hline $\mathbf{T}_{7}$ & 1.98 & 2.10 & 2.20 & 2.32 & 1.80 & 1.98 & 2.96 & 3.20 & 3.30 & 3.39 & 3.14 & 3.20 \\
\hline Mean & 1.88 & 2.14 & 2.34 & 2.73 & 3.09 & 1.88 & 3.11 & 3.65 & 3.90 & 3.99 & 3.80 & 3.69 \\
\hline \multicolumn{13}{|c|}{$\begin{array}{l}\mathrm{T}_{1}: \text { Absolute control } \\
\mathrm{T}_{2}: \text { Package of Practice (rec. NPK+FYM) } \\
\mathrm{T}_{3}: 100 \text { per cent rec. } \mathrm{N}, \mathrm{P} \& \mathrm{~K} \text { (no FYM) } \\
\mathrm{T}_{4}: 75 \text { per cent rec. } \mathrm{P}+\text { rec. } \mathrm{N} \& \mathrm{~K} \text { (no FYM) } \\
\mathrm{T}_{5}: 75 \text { per cent rec. } \mathrm{P}+\text { rec. } \mathrm{N} \& \mathrm{~K}+\text { rec. FYM } \\
\mathrm{T}_{6}: 125 \text { per cent rec. } P+\text { rec. } \mathrm{N} \& \mathrm{~K} \text { (no FYM) }\end{array}$} \\
\hline
\end{tabular}


Continuous application of different organic and inorganic sources of nutrients profoundly influences many chemical properties of soil such as organic carbon content, $\mathrm{pH}$, electrical conductivity etc. Increase in organic matter in soil increases the exchange capacity and cation retention, reduces phosphorus fixation and acts as a source and sink of trace elements resulting in higher availability of nutrients may help in enhancing higher yields. Muralidharudu et al., (2003), Niazi et al., (1990) and Khan et al., (2000) reported increased grain yield due to $\mathrm{P}$ application. Guo Jianhua et al., (2000) observed the residual effects of $\mathrm{P}$ application to wheat increased the succeeding corn yield with 150 $\mathrm{kg} \mathrm{P}_{2} \mathrm{O}_{5}$ ha $^{-1}$ compared to no $\mathrm{P}$, which confirms the importance of P. Wakene Negassa et al., (2005) observed significant residual effects of FYM and NP fertilizers on maize grain yields due to considerable amounts of macronutrients and small amounts of micronutrients which were supplied by FYM.

Among the treatments, application of $125 \%$ rec. $\mathrm{P}+$ rec. $\mathrm{N} \& \mathrm{~K}+$ rec. $\mathrm{FYM}\left(\mathrm{T}_{7}\right)$ recorded significantly higher grain yield. Stover yield was higher due to application of $75 \%$ rec. $\mathrm{P}+$ rec. $\mathrm{N} \& \mathrm{~K}+$ rec. FYM $\left(\mathrm{T}_{5}\right)$ followed by nutrients applied as per package of practice which may be due to the improved physicochemical properties of soil and created an ideal rhizospheric environment which resulted in congenial soil-water relations for better nutrient release and availability besides supplying major and micronutrients.

Sankaran et al., (2005) observed similar results. Interaction effects were higher in $\mathrm{P}_{3}$ strip applied with $125 \%$ rec. $\mathrm{P}+$ rec. $\mathrm{N} \& \mathrm{~K}+$ rec. FYM $\left(\mathrm{T}_{7}\right)$ in grain and $\mathrm{P}_{4}$ strip applied with $\mathrm{T}_{7}$ in straw which may be ascribed to the adequate availability of nutrients in $\mathrm{P}_{3}$ strip and this facilitated greater partitioning of photosynthates to the ear.
The positive interaction may be due to the beneficial effect of FYM application on the availability of phosphorus to the plants. In addition to phosphorus, which was mineralised from the organic matter and became available to plants, organic matter also acts as chelating agents, thereby preventing the formation of insoluble phosphates. Furthermore, the application of organic matter to soil not only supplies phosphorus, but on decomposition provides acidic compounds which increase the availability of mineral forms of phosphorus (i.e., extra $\mathrm{CO}_{2}$ speeded up the solubility of phosphatic compounds) in the soil. Saad Abdel Rahman Sulieman and Tag El-Din Hagoin (2007) reported the similar findings. Lower yield obtained in very low gradient strip with absolute control $\left(\mathrm{T}_{1}\right)$ may be due to supply of lower quantity of nutrients. Kogbe and Adediran (2003) reported the optimum rate of $\mathrm{P}$ for maize grown in the derived savanna was $40 \mathrm{~kg} \mathrm{ha}^{-1}$.

The literature on FYM application to crops clearly indicates that application of FYM increases the microbial activity in soil and resulted in improving the physical properties of soil (viz., structural status, water retention, bulk density of soil) which are reflected on getting higher grain, straw and biomass yields. This could be due to higher availability of nutrients over no FYM application. Combined application of FYM with inorganic fertilizers further enhanced the yield and improved the soil fertility status which resulted in higher crop yield. Sajid Ali et al., (2008) observed increase in maize grain yield with the increase in levels of $\mathrm{P}$ application $\left(60 \mathrm{~kg} \mathrm{ha}^{-1}, 90 \mathrm{~kg} \mathrm{ha}^{-1}\right.$ and $120 \mathrm{~kg}$ $\mathrm{ha}^{-1}$ ) as compared to control and $\mathrm{P}$ dose of 60 $\mathrm{kg} \mathrm{ha}^{-1}$ resulted in maximum increase in grain yield. Sawargaonkar et al., (2008) reported that recommended dose of fertilizer at the rate of 100 and 125 per cent recorded similar mean yield of maize grain and were 
significantly higher over 75 per cent RDF in maize-maize sequence. Venkatesh et al., (2002) noticed that application of $P$ levels upto $60 \mathrm{~kg} \mathrm{ha}^{-1}$ increased grain as well as stover yield of maize significantly as compared to control and $30 \mathrm{~kg} \mathrm{P}_{2} \mathrm{O}_{5} \mathrm{ha}^{-1}$.

Similar yields were obtained with the application of 100 and 125 per cent recommended dose of $\mathrm{P}_{2} \mathrm{O}_{5} \mathrm{ha}^{-1}$. Similar responses were noticed when FYM was applied with different levels of phosphorus. Suryavanshi et al., (2008) noticed that the maize crop responded quadratically to the inputs of nitrogen and phosphorus. Sahoo and Panda (2001) reported the similar results. Balai et al., (2011) obtained maximum grain and stover yield of maize by conjoint application of FYM with recommended dose of NPK over unfertilized control. Damodar Reddy et al., (1999) reported that the integrated use of manure and fertilizer $\mathrm{P}$ is a promising strategy to improve soil $\mathrm{P}$ fertility status and to obtain higher and sustained productivity of the soybean-wheat cropping system.

\section{Harvest Index (HI)}

Harvest index across the $\mathrm{P}$ strips and treatments showed almost similar values except absolute control (Table 3). This could be due to similarity in biological yield though there was a difference in grain and straw/stover yield. Higher grain yield in comparison to stover yield of maize in high gradient strip recorded higher harvest index among the gradient strips. Higher value recorded in medium gradient with the treatment $\mathrm{T}_{7}$ which received $125 \%$ rec. $\mathrm{P}+$ rec. N\&K + rec. FYM followed by high gradient strip with $\mathrm{T}_{7}$ may be due to higher grain yield. Very low gradient with absolute control showed lower HI could be due to lower grain as well as biomass yield. Grain yield and harvest index of cropping system as a whole improved significantly in FYM supplied plots. Anil Choudhary et al., (2007) observed significant improvement in harvest index of wheat and rice crops with increase in chemical fertilizer levels from 50 to 150 per cent of recommended NPK.

The HI was decreased significantly with increased $\mathrm{P}$ fertility gradient strips as well as $P$ levels and was unstable across $P$ levels may be due to genotypic stability for HI was maintained. Reddy et al., (2004) reported similar results. Ti-Da Ge et al., (2012) observed $\mathrm{HI}$ for maize was ranging from 0.20 to 0.40. Dustin et al., (2012) reported harvest index for maize averaged across treatments was 0.49 and none of the treatments were significantly different compared to the control.

\section{P Use Efficiency (PUE)}

Phosphorus use efficiency in maize was higher than finger millet (Table 4) since the maize was known to be exhaustive in nature and also due to residual effect of manures and fertilizers. Generally, lower the P application rate better was the use efficiency. PUE decreased as the fertility gradient increased from very low to very high in finger millet. The higher use efficiency was recorded in very low gradient strip. This may be due to higher response of finger millet to applied $\mathrm{P}$ in very low and low $\mathrm{P}$ gradient strip resulted in to better utilization of native as well as applied P. In case of maize, PUE was higher in high gradient strip followed by medium and low gradient strips due to higher growth of crop and better uptake of $\mathrm{P}$ in high gradient strip. Reduction in PUE in very high gradient strip shows the inefficiency of crop to utilize $\mathrm{P}$ due to adverse effect of higher amount of $\mathrm{P}$ application which affected the crop growth and development which has reduced movement and uptake of nutrients resulting in lower fertilizer P use efficiency. 
Higher PUE among treatments was obtained when $\mathrm{P}$ was applied at the rate of $75 \%$ of recommended dose along with rec. N\&K. This could be due to maximum utilization and uptake of added $\mathrm{P}$ and further application beyond this level was non beneficial and was also non economical to farmers as the PUE by the crops were only 20-40 per cent in general, the remaining amount was fixed in the soil. Application of $\mathrm{P}$ upto $50 \quad \mathrm{~kg} \quad \mathrm{P}_{2} \mathrm{O}_{5}$ ha $^{-1}$ with or without FYM was increased phosphorus use efficiency thereafter it significantly reduced indicating PUE decreases at higher doses of $\mathrm{P}$ due to the fact that plants grown in extremely $\mathrm{P}$ deficient soil exhibit greater $\mathrm{P}$ sorption at lower doses of $\mathrm{P}$. In areas of marginal $\mathrm{P}$ deficiency, application of $\mathrm{P}$ fertilizers is essential to marginal higher yields under intensive cropping. Nutrient use efficiency and productive efficiency decreased with increasing doses of $P$ application. Venkatesh et al., (2002) reported similar results.

Bisht and Chandel (1991) noticed the higher photosynthetic rate and physiological efficiency of nitrogen was obtained with the application of $80 \mathrm{~kg} \mathrm{P}_{2} \mathrm{O}_{5}+10 \mathrm{t} F Y M$ per ha. Majumdhar et al., (2007) observed the higher $\mathrm{P}$ use efficiency at lower doses of applied $\mathrm{P}$ and maximum was 21.30 per cent recorded with SSP @ 30 kg $\mathrm{P}_{2} \mathrm{O}_{5} \mathrm{ha}^{-1}+$ FYM. Dhillon et al., (1987) reported that the $\mathrm{P}$ use efficiency by pearl millet was maximum in low $\mathrm{P}$ soils and decreased with increase in available $\mathrm{P}$ status. Further, the increasing level of applied $\mathrm{P}$ also resulted in low $\mathrm{P}$ utilization and hence, fertilizer efficiency decreased. Datta and Gomez (1975) reported an increase in $P$ use efficiency with successive cropping's. Muralidharudu et al., (2003) obtained relatively higher NUE and $\mathrm{PE}$ in low and high available $\mathrm{P}$ soils due to application of $30 \mathrm{~kg} \mathrm{P}_{2} \mathrm{O}_{5} \mathrm{ha}^{-1}$. Apparent $\mathrm{P}$ recovery declined with increasing rates of $P$ application.

\section{B: C ratio}

Higher $\mathrm{B}$ : $\mathrm{C}$ ratio in finger millet and maize were recorded in very high and high $\mathrm{P}$ fertility gradient strip could be due to higher yield obtained due to residual as well as added fertilizer effect (Table 5). Application of FYM along with inorganics recorded lower B: $\mathrm{C}$ ratio due to higher cost of FYM purchase resulting higher cost of cultivation and lower $B$ : $C$ ratio. If the manure produced by the farmer the cost of cultivation reduces and sustains $\mathrm{B}$ : $\mathrm{C}$ ratio. In case of maize there was a slight decrease in $\mathrm{B}$ : $\mathrm{C}$ ratio in very high gradient could be due to lower yield resulting in lower gross and net income. Among treatments, application of $125 \%$ rec. $\mathrm{P}+$ rec. $\mathrm{N} \& \mathrm{~K}\left(\mathrm{~T}_{6}\right)$ and $75 \%$ rec. $\mathrm{P}+$ rec. $\mathrm{N} \& \mathrm{~K}\left(\mathrm{~T}_{4}\right)$ noticed higher $\mathrm{B}$ : $\mathrm{C}$ ratio may be due to better yield and lower cost of inputs as the nonaddition of manure.

Application of $75 \%$ rec. $\mathrm{P}+$ rec. N\&K recorded higher $\mathrm{B}$ : $\mathrm{C}$ ratio in both the crops and was the best and optimum $\mathrm{P}$ prescription for these crops as the $\mathrm{P}$ use efficiency was also higher. Application of higher dose of $\mathrm{P}$ fertilize has no beneficial effect in high and very high $\mathrm{P}$ fertility strips. Very low gradient with absolute control showed lower benefit cost ratio which indicates that application of sufficient amount of nutrients was very much essential in order to achieve better crop yields and income. There was an increased B: C ratio in absolute control with increased $P$ fertility gradients there was increase in yield which shows the role and importance of phosphorus for crop production. Similar findings were reported by Vinodkumar (2008), Rajput et al., (1995), Singh et al., (1999), Singh and Verma (2002) and Balasubramaniyan (2005).

It was concluded that, application of fertilizer P @ $125 \%$ RDP with rec. NK\&FYM in very low, low and medium fertility soils recorded 
higher grain and straw/stover yield of finger millet and maize. $100 \%$ or $75 \%$ RDP with rec. NK\&FYM were optimum though there was an increase in yield at higher rate of $\mathrm{P}$ application in high $\mathrm{P}$ fertility soils. PUE was higher at lower levels of $\mathrm{P}$ application.

\section{Acknowledgments}

I would like to thank chairman and members of advisory committee, staff's of Dept. of SS\&AC, UAS, Bangalore, NBSS\&LUP and ICAR and my beloved family members and friends. Thankful to ICAR, New Delhi for granting SRF and RKVY, Govt. of Karnataka for providing financial assistance for conducting research.

\section{References}

Ali, M. I. and Rahman, G. K. M. M., 2011, Phosphorus extractability in Bangladesh soils and its critical limit for rice and wheat. Bangladesh Institute of Nuclear Agriculture, P.O. Box 4, Mymensingh, Bangladesh.

Ananda, N., 2009, Relative response of finger millet-soybean rotation to application of zinc, boron and microbial inoculants, Ph.D. Thesis, University of Agricultural Sciences, GKVK, Bangalore, Karnataka, India.

Anil Choudhary, K., Thakur, R. C. and Naveen Kumar, 2007, Effects of organic manures and chemical fertilizers on productivity and profitability in wheat - rice cropping sequence, Oryza, 44(3): 239-242.

Anonymous, 1996, Annual report, Long term fertilizer experiment (LTFE), UAS Bangalore, Karnataka, India., pp: 1-30

Anonymous, 2000, Annual progress report, All India Co-ordinated Research Project for Dryland Agriculture, UAS, Bangalore, Karnataka, India.

Anonymous, 2009, Results of front line demonstrations (2000-2007) and technologies for increasing small millet production in India, Project coordinating unit (small millets), ICAR, Bangalore.
Anonymous, 2012, Effect of long-term fertilizer and manure application on Pfractionation in Alfisols of Bangalore. Indian Institute of Soil Science. Bhopal, http://www.iiss.nic.in/Rnd.htm.

Balai, M. L., Arvind Verma, Nepalia, V. and Kanthaliya, P. C., 2011, Productivity and quality of maize (Zea mays) as influenced by integrated nutrient management under continuous cropping and fertilization. Indian J. Agric. Sci., 81(4): 374- 376.

Balasubramaniyan, P., 2005, Integrated use of organics and phosphorus in pre monsoon direct seeded rainfed un-puddled lowland rice. Madras Agric. J., $\quad 92(10-12)$ : 680683.

Bisht, J. K. and Chandel, A. S., 1991, Effect of INM on leaf area index, photosynthetic rate, agronomic and physiological efficiencies of soybean. Indian J. Agron., 36: 129-132.

By Guo Jianhua, Xing Zhu and Liu Zongheng, 2000, Responses to phosphorus and potassium application in a wheat-corn rotation in Hebei Province. Better Crops Int., 14 (2): 3-5.

Damodar Reddy, D., Subba Rao, A., Sammi Reddy, K., Takkar, P. N., 1999, Yield sustainability and phosphorus utilization in soybean-wheat system on Vertisols in response to integrated use of manure and fertilizer phosphorus. Field Crops Res., 62: 181-190.

De Datta, S. K. and Gomez, K. A., 1975, changes in soil fertility under intensive rice cropping with improved varieties. Soil Sci. Soc. America proc., 120(5): 361366.

Dhillon, N. S., Vig, A. C., Milap Chand, Bhan Singh and Dey, G., 1987, Critical level of phosphorous in soils of Punjab and response of pearl millet to applied phosphate. J. Indian Soc. Soil Sci., 35: 238-43.

Dustin, R., Wiggans Jeremy, W., Singer Kenneth, J., Moore and Kendall, R., Lamkey, 2012, Response of continuous maize with stover removal to living mulches. Agron. J., 104 (4): 917-925. 
Ghanshyam, Rakesh Kumar and Jat, R. K., 2010, Productivity and soil fertility as effected by organic manures and inorganic fertilizers in greengram (Vigna radiata) - wheat (Triticum aestivum) system. Indian J. Agron., 55(1): 16-21.

Hanumanthappa, M. and Shivaraj, B., 2003, Effect of organic manures and fertilizer levels on yield, components and nutrient uptake of sesamum under rainfed conditions. Mysore J. Agric. Sci., 37(2): 144-151.

Khalil, I. A. and Jan, A., 2003, Cropping Technology. National Book Foundation, Islamabad, Pakistan.

Khan, A., Azam, M. and Khan, M., 2000, Grain yield and economic effect of NP fertilizers application on dryland barley. Pakistan J. Biol. Sci., 3: 816-818.

Kogbe, J. O. S. and Adediran, J. A., 2003, Influence of nitrogen, phosphorus and potassium application on the yield of maize in the savanna zone of Nigeria, African J. Biotech., 2(10): 345-349.

Lu Ru-Kun, 1981, The fertility and fertilizer use of important paddy soils. In Proceedings of the Symposium on Paddy Soils (Ed.), Institute of Soil Science, Academia Simica, Science Press, Beijing., pp: 160170.

Majumdhar, B., Venkatesh, M. S., Kailash Kumar and Patiram, 2007, Effect of rock phosphate, superphosphate and their mixtures with FYM on soybean and soil$P$ pools in a Typic Hapludalf of Meghalaya. J. Indian Soc. Soil Sci., 55(2): 167-174.

Moll, R. H., Kamprath, E. J. and Jackson. W. A., 1982. Analysis and interpretation of factors which contribute to efficiency of nitrogen utilization. Agron. J., 74: 562564.

More, S. D., and Agale, B. N., 1993, Phosphate balance studies in irrigated cotton. J. Indian Soc. Soil Sci., 41(3): 498-500.

Muralidharudu, Y., Murthy, I. Y. L. N., Reddy, K.P. C., Reddy, B. N. AND Chandranath, H. T., 2003, Response of sunflower (Helianthus annuus L.) to phosphorus application in Vertisols. HELIA, UDC 633.854.78:631.416.2, 26(39): 147-154.

Niazi, M. H. K., Mehdi, S. M. and Iqbal, J., 1990, Phosphorus requirement of rice and wheat in saline-sodic and sodic soils. Role of phosphorus in crop production. NFDC, Islamabad, Pakistan., pp: 439449.

Nimje, P.M. and Seth, J., 1988, Effect of phosphorus and FYM on nutrient uptake by soybean. Indian J. Agron., 33 (2): 139142.

Panse, V. G. and Sukhatme, P. V., 1985, Statistical methods for agricultural worker. Publication and information division, ICAR, New Delhi.

Prasad, B. and Prasad, J., 1994, Integrated nutrient management for specific yield of rice based on targeted yield concept and soil test values in old alluvial soils. Oryza., 31: 140-143.

Prasad, B., Sinha, R. and Prasad, J., 1984, Fertilizer requirement for different yield target of chewing tobacco based on soil test values in a calcareous soil of Bihar. Tobacco Res., 10(2): 176-181.

Rajput, A. M., Shroff, V. N. and Deshpande, S., 1995, Impact of mixed organic farming (organic manure + fertilizer) on soybean and potato. Crop Res., 10 (3): 258-260.

Ramamoorthy, B., Narasimham, R. L and Dinesh, R. S., 1967, Fertilizer application for specific yield target of sonara-64 wheat. Indian Farm., 17: 43-45.

Reddy, D. V. V., Udayakumar, M., Prasad, T. G., Seethram, A. and Nanjareddy, Y. A., 2004, Influence of NPK on relative stability of harvest index in finger millet. Karnataka J. Agric. Sci., 17(4): 691-695.

Saad Abdel Rahman Sulieman and Tag El-Din Hago, E. M., 2007, The Response of Common Bean (Phaseolus vulgaris L.) to Phosphorus as Influenced by farmyard manure. Univ. Khartoum, Faculty of Agric., Sudan.

Sahoo, S. C., and Panda, M. M., 2001, Effect of phosphorus on yield of baby corn. Indian J. Agric. Sci., 71(1): 21-22. 
Sajid Ali, A., Riaz Khan, Ghazal Mairaj, Arif, M., Mehwish Fida and Saiqa Bibi, 2008, Assessment of different crop nutrient management practices for yield improvement, Australian J. Crop Sci., 2(3): 150-157.

Sawargaonkar, G. L., Shelke, D. K. and Shinde, S. A., 2008, Influence of cropping systems and fertilizer doses on dry matter accumulation and nutrient uptake by maize (Zea mays L.). Int. J. Agric. Sci., 4(1): 45-50.

Singh, N. B. and Verma, K. K., 2002, Response of French bean to application of organics and inorganics in eastern Uttar Pradesh. Indian J. Agron., 47: 81-85.

Singh, N. P., Sachan, R. S., Pande, P. C. and Bisht, P. S., 1999, Effect of decaded long fertilizer and manure application on soil fertility and productivity of ricewheat system in Mollisol. J. Indian Soc. Soil Sci., 47(1): 72-80.

Subramanian, K. S. and Kumaraswamy, K., 1989, Effect of continuous cropping and fertilization on chemical properties of soil. J. Indian Soc. Soil Sci., 37: 171-173.

Suryavanshi, V. P., Chavan, B. N., Jadhav, V. T. and Baig, M. I. A., 2008, Response of maize to nitrogen and phosphorous application in Vertisols. Int. J. Trop. Agric., 26: 293-296.

Terao, T. K, Nagata, K. and Hirose, T., 2011, A gene controlling the number of primary rachis branches also controls the vascular bundle formation and hence is responsible to increase the harvest index and grain yield in rice. Theor. Appl. Genet., 120: 875-893.

Thimma Reddy, 1987, Phosphorus fixation in Alfisol of Bangalore as influenced by permanent manuring and cropping schedule and its influence on yield of finger millet. M.Sc. (Agri.) Thesis, UAS, Bangalore, Karnataka, India.

Ti-Da GE, Ning-Bo Sun, Li-Ping Bai, Chen-Li Tong and Fang-Gong Sui, 2012, Effects of drought stress on phosphorus and potassium uptake dynamics in summer maize (Zea mays) throughout the growth cycle. Acta. Phys. Plant, 10.1007/s11738012-1018-7.

Tiwari, K. N., 2002, Fertilizer recommendations: need a fresh look. Research Education, Fertilizer knowledge, 4.

Venkatesh, M. S., Majumdar, B., Kailash kumar and Patiram, 2002, Effect of phosphorous, FYM and lime on yield, $P$ uptake by maize and forms of soil acidity in Typic hapludalf of Meghalaya. J. Indian Soc. Soil Sci., 50(3): 254-258.

Vinod Kumar, R., 2008, Soil-site suitability for finger millet on watershed approach at varied fertility levels, M.Sc. (Agric), Thesis, UAS, Bangalore, Karnataka, India.

Virupakshi, B. E., 1988, Available micronutrients and yield of finger millet (Eleusine coracana (L.) Gaertn.) in Alfisols of Bangalore as influenced by intensive manure and cropping sequence., M.Sc. (Agri.) Thesis, UAS, Bangalore, Karnataka, India.

Wakene Negassa, Heluf Gebrekidan and Friesen, D. K., 2005, Integrated use of farmyard manure and NP fertilizers for maize on farmers' fields. J. Agri. Rural Dev. Tropics and Subtropics, 106(2): 131-141.

Zhang Xiao-Sheng, Liao Hong, Chen Qing1y, P., Christie1g, L. I., Xiao-Lin and Zhang Fu-Suo, 2007, Response of Tomato on Calcareous Soils to Different Seedbed Phosphorus Application Rates. Pedosphere, 17(1): 70-76.

\section{How to cite this article:}

Chandrakala, M., C.A. Srinivasamurthy, Sanjeev Kumar and Naveen, D.V. 2017. Effect of Application of Graded Level of Phosphorus to Finger Millet - Maize Cropping System in Soils of Different P Fertility. Int.J.Curr.Microbiol.App.Sci. 6(11): 265-280.

doi: https://doi.org/10.20546/ijcmas.2017.611.032 